034.

\section{Hand-Washing Compliance Among Emergency Medical Responders}

T. Chadwick Eustis, EMT, * Elisabeth J. Fowlie, EMT, Seth W. Wright, MD, FACEP, Keith D. Wrenn, MD, FACEP, Corey $M$. Slovis, $M D, P h D$

Vanderbilt University School of Medicine, Department of Emergency Medicine, Nashville, Tennessee USA

Purpose: To evaluate the compliance of emergency medical responders (EMRs) with federal and local recommendations for hand washing.

Methods: Prospective, single-blinded, observational study of 68 EMRs during 258 ambulance responses over a three-month period. Two medical students observed the EMRs of an urban emergency medical services (EMS) system. The EMRs were blinded to the existence of this study. Observers recorded EMRs' glove use during patient contacts and ambulance cleaning, as well as subsequent hand washing before returning to service.

Results: Of 457 observed patient contact or cleaning events, hands were washed $383(84 \%)$ times. Thirty-six $(53 \%)$ of EMRs were responsible for the observed hand-washing violations. In 441 patient contacts, EMRs wore protective gloves 363 (79\%) times and washed their hands $371(84 \%)$ times. EMRs washed their hands $311(86 \%)$ times after the 363 gloved contacts, but only washed $60(77 \%)$ times after 78 ungloved contacts $(p=0.06)$. In 192 cleanings, EMRs wore gloves $115(60 \%)$ times, and washed their hands $170(89 \%)$ times. EMRs washed their hands $110(96 \%)$ times after the 115 gloved cleanings, but only washed $60(78 \%)$ times after 78 ungloved cleanings $(p=0.0002)$. Conclusions: Hand washing is one of the most effective means to limit the spread of infectious diseases to and by health-care workers. EMRs do not universally follow recommendations for hand washing after every patient contact or cleaning of patient-care areas. Surprisingly, hand washing was less frequent if gloves were not worn, thus, further increasing the risk of disease transmission.
010.

Defective Color Vision in Paramedics and Its Effect on Blood Glucose Test Strip Interpretation

Jonathan M. Rubin, $M D,{ }^{*}$ Ronald G. Pirrallo, $M D, M H S A$

Department of Emergency Medicine, Medical College

of Wisconsin, Milwaukee, Wisconsin USA

Objectives: Paramedics commonly perform blood glucose testing based on the color change of a reagent. A paramedic with defective color vision may misinterpret this test and treat a patient inappropriately. Our goal was to determine both the incidence of color vision deficiency in paramedics in our EMS system and its effect on the interpretation of a semi-quantitative blood glucose test strip.

Methods: All paramedics attending a quarterly meeting participated in this two-part study. The first part of the study consisted of standardized color vision testing under natural light conditions using 11 Ishihara plates. The second part of the study entailed reading two pre-prepared blood glucose test strips (Chemstrip $\left.b^{(}\right)$). The testing strips were made by cutting out the reference color blocks corresponding to 80 $\mathrm{mg} / \mathrm{dL}$ and $240 \mathrm{mg} / \mathrm{dL}$ from the chart on the Chemstrip bG ${ }^{\circledR}$ bottle and affixing one block to the end of a strip. Without knowing the results of their color vision testing, the paramedics then interpreted the two strips by comparing them with the reference blocks on the bottle.

Results: Defective color vision was found in seven of the 122 $(5.7 \%)$ paramedics tested. Five of 102 males $(4.9 \%)$ and two of 20 females $(10 \%)$ were found to be color deficient. None of the color-deficient paramedics misread either test strip. Of those with normal color vision, 14 of $115(12.2 \%)$ misread the first strip, and 10 of $115(8.7 \%)$ misread the second. None of the incorrect readings was more than one increment away from the correct value, and all were higher than the actual value.

Conclusions: Paramedics with defective color vision in our EMS system are able to interpret semi-quantitative blood glucose test strips accurately. The error rate in those with normal color vision may be important in clinical practice. 